Alex Paúl Zambrano-Álava; Ana Thalía Lucas-Zambrano; María De Los Ángeles Lucas-Zambrano; Karina Elizabeth Luque-Alcívar

http://dx.doi.org/10.35381/e.k.v3i5.847

\title{
Gamificación y aprendizaje autorregulado
}

\section{Gamification and self-regulated learning}

\author{
Alex Paúl Zambrano-Álava \\ paul.zambrano.1990@gmail.com \\ Pontificia Universidad Católica del Ecuador, Sede Manabí, Campus Chone \\ Ecuador \\ https://orcid.org/0000-0001-9327-6353 \\ Ana Thalía Lucas-Zambrano \\ mdlalucas@gmail.com \\ Pontificia Universidad Católica del Ecuador, Sede Manabí, Campus Chone \\ Ecuador \\ https://orcid.org/0000-0002-1214-3477 \\ María De Los Ángeles Lucas-Zambrano \\ kaellual@gmail.com \\ Pontificia Universidad Católica del Ecuador, Sede Manabí, Campus Chone \\ Ecuador \\ https://orcid.org/0000-0001-8253-6287 \\ Karina Elizabeth Luque-Alcívar \\ anathalialucas@gmail.com \\ Pontificia Universidad Católica del Ecuador, Sede Manabí, Campus Chone \\ Ecuador \\ https://orcid.org/0000-0002-2052-1087
}

Recepción: 20 abril 2020

Revisado: 25 de mayo 2020

Aprobación: 20 junio 2020

Publicación: 26 de junio 2020 
Alex Paúl Zambrano-Álava; Ana Thalía Lucas-Zambrano; María De Los Ángeles Lucas-Zambrano; Karina Elizabeth Luque-Alcívar

\title{
RESUMEN
}

Con la investigación se planteó determinar la relación entre gamificación y aprendizaje autorregulado en estudiantes de educación básica general de la provincia de ManabíEcuador. El estudio es de tipo correlacional, con diseño no experimental transversal. Se trabajó con una población conformada por 70 docentes de la ciudad de Chone, a quienes se les aplicó dos instrumentos estandarizados. Considerando la muestra investigada y los resultados obtenidos, se tiene que el coeficiente de correlación de Pearson es de 0,104 . Esto indica que existe relación positiva baja entre las variables de estudio, lo que implica que a mayor uso de la gamificación mayor autorregulación en el proceso de aprendizaje. En consecuencia, se determina que la gamificación se relaciona positivamente con el aprendizaje autorregulado, lo cual favorece al estudiante en su motivación y crecimiento personal como factores esenciales para promover un mejor rendimiento académico, desde un proceso centrado en el estudiante.

Descriptores: Juego educativo; material didáctico; programa informático didáctico; enseñanza asistida por ordenador. (Palabras tomadas del Tesauro UNESCO).

\begin{abstract}
With the investigation, it was proposed to determine the relationship between gamification and self-regulated learning in students of general basic education in the province of Manabí-Ecuador. The study was correlational, with a non-experimental cross-sectional design. The researchers worked with a population made up of 70 teachers from the city of Chone, to whom two standardized instruments were applied. Considering the investigated sample and the results obtained, the Pearson's correlation coefficient was 0.104 . This indicates that there is a low positive relationship between the study variables, which implies that the greater the use of gamification, the greater self-regulation in the students' learning process. Consequently, it is determined that gamification is positively related to self-regulated learning, which favors the students' motivation and personal growth as essential factors to promote better academic performance, from a studentcentered process.
\end{abstract}

Descriptors: Educational games; teaching materials; educational software; computer assisted instruction. (Words taken from the UNESCO Thesaurus). 
Alex Paúl Zambrano-Álava; Ana Thalía Lucas-Zambrano; María De Los Ángeles Lucas-Zambrano; Karina Elizabeth Luque-Alcívar

\section{INTRODUCCIÓN}

En un mundo digitalizado el acceso a la información es continuo y se encuentra al alcance de todos, la experiencia que generan las nuevas tecnologías en los usuarios es sumamente enriquecedora, por cuanto los transporta a otra realidad, tal es el caso de los videojuegos, los cuales captan el interés del usuario, esto quizás porque el ser humano se encuentra relacionado con el juego (Valda-Sánchez \& Arteaga-Rivero, 2015). Es así que la educación ha innovado al incorporar juegos digitales en la generación de aprendizaje, bien sea como estrategia, recurso o herramienta, siendo una tendencia la gamificación por cuanto promueve la capacidad cognitiva de los estudiantes (LiberioAmbuisaca, 2019).

En este sentido, la gamificación promueve la transformación educativa en sintonía a la motivación de los estudiantes, por cuanto al asociar el juego con el aprendizaje, el discente genera expectativas favorables de apertura a las instrucciones del docente con la finalidad de cumplir con las metas propuestas o diseñadas en el plan educativo, siendo indispensable el acompañamiento de los padres y representantes, por cuanto el estudiante en sus inicios debe ser asesorado hasta lograr un aprendizaje autónomo (Area-Moreira \& González-González, 2015), es evidente que los estudiantes alcanzan un gran nivel de compromiso cuando se encuentran motivados, incluso prefiriendo seguir con la actividad lúdica a dar por finalizada la clase (Fernández, Olmos\& Alegre, 2016).

Una de las bondades de la gamificación es que minimiza el aburrimiento a la clase, genera mayor interés en el estudiante, representando así una estrategia significativa en el logro de los objetivos de aprendizaje, siendo estimulante en los estudiantes para realizar interacciones con el aprendizaje desde una perspectiva social y colaborativa, transcendiendo el vacío fomentado desde la educación tradicional donde el discente puede percibirse como aislado para la realización de diversas tareas, así como también incentiva la motivación como elemento fundamental para concretar un proceso favorable 
Alex Paúl Zambrano-Álava; Ana Thalía Lucas-Zambrano; María De Los Ángeles Lucas-Zambrano; Karina Elizabeth Luque-Alcívar

al logro de los propósitos planificados en el cronograma escolar (Ortiz-Colón, et al., 2018). Es así que la gamificación ante la falta de interés hacia el aprendizaje por parte de los estudiantes, se constituye en una estrategia o herramienta favorable para promoverlo (Zepeda-Hernández, et al., 2017). Además, la gamificación mediante los videos juegos, se da como medio efectivo para la consecución de un aprendizaje significativo en los estudiantes (Hernández-Horta, et al., 2018), al estos concebir la autoobservación, reflexión, como procesos de un aprendizaje autorregulado que propicia la generación de la autonomía para asumir responsablemente sus actividades inherentes a la planificación educativa (Torrano, et al., 2017).

El aprendizaje autorregulado se ha destacado potencialmente en los últimos años por sus resultados en aprendizajes autónomos como pieza clave en el discente; pues esto le ha generado mayor capacidad de adaptación a cualquier circunstancia académica, mas independencia y mayor motivación para aprender (Torrano, Fuentes \& Soria, 2017). En este tenor, el aprendizaje autorregulado puede contribuir en el diseño de planes individualizados como medio para propiciar la transcendencia de debilidades en el estudiante en función de lograr la efectividad en el rendimiento académico (MedinaRamírez, et al., 2019).

En función de lo abordado, la investigación tiene por objetivo determinar la relación que existe entre gamificación y aprendizaje autorregulado en estudiantes de educación básica general de la provincia de Manabí-Ecuador. Esto con la finalidad de conocer como se está desarrollando este proceso a partir de la aplicación de estrategias por parte de los docentes.

\section{MÉTODO}

La investigación es de tipo correlacional, con diseño de campo, no experimental transversal (Hernández-Sampieri, et al., 2014). Siendo la población 70 docentes de la ciudad de Chone, provincia de Manabí-Ecuador a quienes se les aplicó dos instrumentos 
Alex Paúl Zambrano-Álava; Ana Thalía Lucas-Zambrano; María De Los Ángeles Lucas-Zambrano; Karina Elizabeth Luque-Alcívar

estandarizados para medir las variables gamificación y aprendizaje autorregulado. En este sentido, para la gamificación se empleó el instrumento validado por (Parra-González, et al., 2019), mientras que para el aprendizaje autorregulado, se empleó el propuesto por (Núñez, et al., 2019). Para el cálculo de la correlación entre las variables estudiadas se consideró el Coeficiente de Correlación de Pearson; asimismo, los datos obtenidos se procesaron mediante la estadística descriptiva empleando para ello el programa Spss V25.

\section{RESULTADOS}

\section{Tabla 1 - Gamificación}

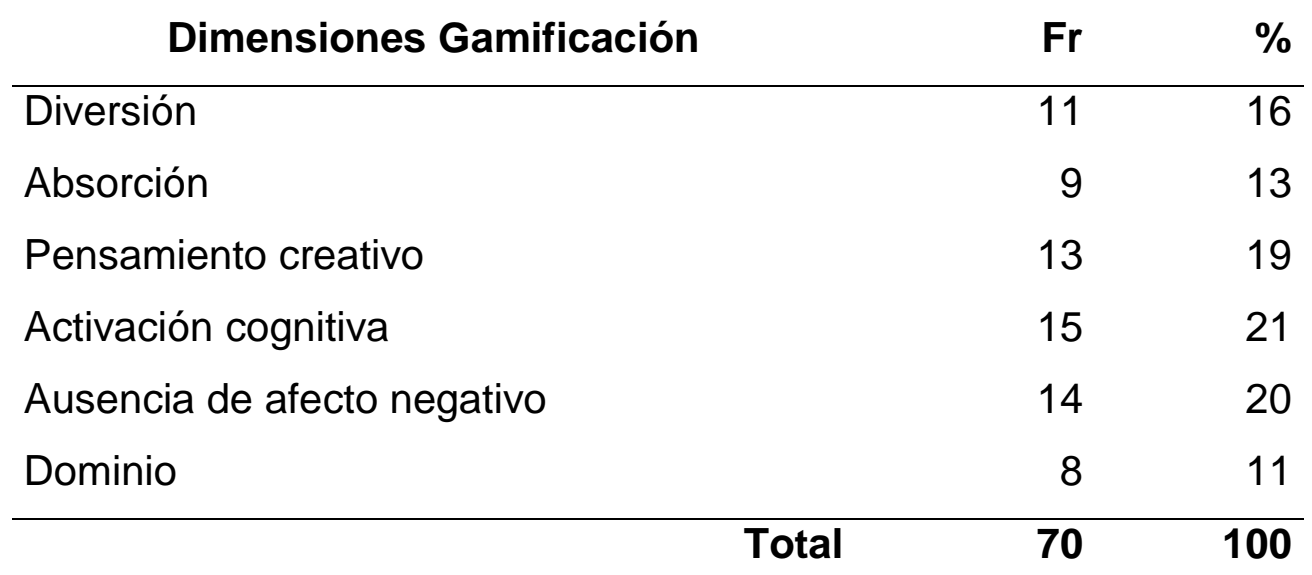

Las dimensiones de gamificación con mayor énfasis porcentual, se destaca activación con $21 \%$, ausencia de afecto negativo $20 \%$ y pensamiento creativo con $19 \%$ como las de mayor frecuencia por parte de la población encuestada. 
Alex Paúl Zambrano-Álava; Ana Thalía Lucas-Zambrano; María De Los Ángeles Lucas-Zambrano; Karina Elizabeth Luque-Alcívar

\section{Tabla 2}

Aprendizaje autorregulado

\begin{tabular}{|c|c|c|}
\hline Dimensiones & $\mathrm{Fr}$ & $\%$ \\
\hline Planificación & 12 & 17 \\
\hline Gestión de la cognición & 13 & 19 \\
\hline Gestión de la motivación & 16 & 23 \\
\hline Evaluación de la comprensión & 14 & 20 \\
\hline Gestión del contexto & 15 & 21 \\
\hline & 70 & 100 \\
\hline
\end{tabular}

En el aprendizaje autorregulado se destaca la gestión de la motivación como la de mayor porcentaje (23\%), destacándose el $20 \%$ de la evaluación de la comprensión y $21 \%$ relacionado a la gestión del contexto, como las dimensiones de mayor alcance por parte de la población encuestada.

Tabla 3 - Relación gamificación y aprendizaje autorregulado

\section{Correlaciones}

\begin{tabular}{llrr} 
& & DG & \multicolumn{2}{c}{ AA } \\
\hline DG & Correlación de Pearson & 1 & 0,104 \\
& Sig. (bilateral) & & 0,392 \\
& $N$ & 70 & 70 \\
AA & Correlación de Pearson & 0,104 & 1 \\
& Sig. (bilateral) & 0,392 & \\
N & 70 & 70 \\
\hline
\end{tabular}


Alex Paúl Zambrano-Álava; Ana Thalía Lucas-Zambrano; María De Los Ángeles Lucas-Zambrano; Karina Elizabeth Luque-Alcívar

El coeficiente de correlación de Pearson de 0,104 indica que existe relación positiva baja entre las variables de estudio, lo cual implica que la gamificación influye positivamente en la generación del aprendizaje autorregulado en la muestra investigada, se destaca que la correlación al ser mayor a 0,05 no existe significancia bilateral en la correlación.

\section{DISCUSIÓN}

Relacionando el estudio de (Cortez-Godinez, 2017) con los resultados obtenidos, se puede decir que en el aprendizaje gamificado, la diversión se constituye en el estímulo preparatorio para el procesamiento cognitivo de la información y procedimientos a desarrollar para alcanzar una determinada meta propuesta en el plan de estudios. Efectivamente fomenta el aprendizaje; por tanto, es un factor a tener en cuenta por parte de los docentes, padres y representantes, a fin de coordinar acciones favorables a emplear el juego como parte de los estudios académicos.

Asimismo, la absorción del conocimiento de esta manera, se da evidentemente optimizando el rendimiento académico y elevando su estima como estudiante (OleaMiranda, et al., 2016). Por tanto, es favorable propiciar la gamificación como marco para el desarrollo didáctico del aprendizaje, pues esto conlleva a desarrollar ventajas en el estudiante en comparación con quienes no tienen esta habilidad o competencia desarrollada.

Con respecto al pensamiento creativo, la incorporación de la gamificación en el proceso de aprendizaje prepara al estudiante para enfrentar los retos de una sociedad basada en la tecnología, donde la creatividad toma un valor esencial para transcender a la germinación de soluciones en referencia al contexto social donde se desenvuelve; pues como afirman (Hernández-Jaime, et al., 2018), es sumamente importante promover una educación basada en la creatividad, innovación, como pilares fundamentales para lograr cambios significativos en el abordaje de la realidad socio económica de los estudiantes. El aprendizaje gamificado se constituye en un potente activador de la cognición, en el 
Alex Paúl Zambrano-Álava; Ana Thalía Lucas-Zambrano; María De Los Ángeles Lucas-Zambrano; Karina Elizabeth Luque-Alcívar

sentido que despierta el interés del estudiante para desarrollar con eficacia un tema de estudio, situación que le permite realizar procesos mentales en favor de un aprendizaje duradero en el tiempo (Muñoz-González, 2018). El dominio por parte del estudiante de los diversos temas de estudios focalizados en el ambiente de aprendizaje, le permite transitar hacia la consecución de competencias que apuntan a la consecución de un aprendizaje significativo en función de alcanzar metas propuestas en el ámbito escolar, siendo posible trasladar esta postura hacia lo personal y social (Villarroel \& Bruna, 2017). Asimismo, esta activación cognitiva promueve la apertura al contexto social, lo que propicia un abordaje de los contenidos educativos contextualizados a la realidad. Es decir, induce y estimula las operaciones mentales en función de la resolución de problemas reales, lográndose con ello el logro efectivo del propósito de aprendizaje establecido (Martínez-Albán, et al., 2019).

En este contexto, la gamificación propicia la ausencia de afecto negativo. Lo que significa que contribuye tanto al logro de un aprendizaje significativo, como al crecimiento personal del estudiante al favorecer una actitud positiva sobre sí mismo (Mujica-Johnson \& ToroOyarzún, 2019). De ahí que sea importante formar a la familia cercana al estudiante con la finalidad de que comprendan la importancia del juego desde una perspectiva pedagógica, que superen la visión del juego como pérdida de tiempo. Así la familia se convierte en el más importante elemento de apoyo que propicia la autoestima positiva que compone la dimensiona afectiva del estudiante (Hernández, 2018).

Con respecto al aprendizaje autorregulado, su planificación posibilita que los estudiantes organicen y sistematicen la búsqueda de información, generándose así un proceso autónomo que progresivamente conduce al desarrollo de competencia autodidacta en ellos; lo que conlleva, simultáneamente, que el docente asuma cada vez más un enfoque de planificación curricular centrado en el estudiante (Vallejo-Valdivieso, et al., 2019). Visto así, la planeación del se constituye en un potente proceso para el aprendizaje significativo del estudiante. Pues promueve la autogestión del conocimiento tornándolo duradero en 
Alex Paúl Zambrano-Álava; Ana Thalía Lucas-Zambrano; María De Los Ángeles Lucas-Zambrano; Karina Elizabeth Luque-Alcívar

el tiempo, a la vez que contribuye a fomentar una visión organizadora de la gestión de vida en función de percibir el éxito como producto de un proceso y no del azar (MoralesSalas, 2018).

Es de saber, que la gestión de la cognición de esta manera se genera desde la interrelación del estudiante con el contenido abordado mediante la gamificación, lo cual se constituye en un eje transversal que propicia conocimientos con visión multidisciplinar (Rimassa, 2016). Además, este proceso que también se concibe metacognitivo, hace que los estudiantes concienticen sus conocimientos de la realidad y la necesidad de transformarlos continuamente. Por tanto, el aprendizaje autorregulado favorece la construcción de saberes en proposición de solventar problemáticas cotidianas con apoyo del aprendizaje académico como sostiene (Muñoz-Morales, et al., 2019).

Desde el aprendizaje autorregulado, la gestión de la motivación es imprescindible, pues contribuye a que el estudiante alcance las metas planteadas en la planificación educativa; cuando el estudiante consigue organizar los procesos cognitivos y académicos, se percibe motivado y se suscita un aprendizaje significativo (Valenzuela, et al., 2015). De allí que la motivación se constituye en un factor importante y primordial del proceso enseñanza - aprendizaje; pues con ella se promueve el desarrollo de las competencias emocionales de los estudiantes (Alemán-Marichal, et al., 2018), y la integración familiar, dado el acompañamiento que esta debe dar para lograr tal fin de modo efectivo (Páez, 2018).

El aprendizaje autorregulado conlleva a la comprensión del conocimiento y a las formas de producirlo, transcendiendo la enseñanza mecanicista. En este sentido, el estudiante asume el proceso de conocimiento desde una perspectiva global del contexto académico y social donde se desenvuelve, y en favor de generar la resolución de problemas; por lo que se asume un enfoque activo y constructivo del aprendizaje (Castelló \&Cladellas, 2013). 
Alex Paúl Zambrano-Álava; Ana Thalía Lucas-Zambrano; María De Los Ángeles Lucas-Zambrano; Karina Elizabeth Luque-Alcívar

En este mismo contexto, el aprendizaje autorregulado se enfoca en situar al docente y estudiante en el rol de asesor y asesorado como equipo de trabajo para la autorregulación del aprendizaje. Así pues, el rol del estudiante se constituye en centro del proceso (Daura, 2013), proyectándose por medio de la investigación e indagación, como fundamentos pedagógico, para la generación de conocimientos (Peche-Cruz \& Giraldo-Supo, 2019). Situación que coadyuva a la atención psicopedagógica en función de las necesidades e intereses del estudiante, posibilitando un aula inclusiva y de calidad educativa (Zambrano-Pincay, et al., 2019).

Ahora bien, al generarse una correlación positiva baja entre la gamificación y el aprendizaje autorregulado, se considera que se fomenta una motivación favorable en los estudiantes para alcanzar sus metas académicas desde la autorregulación, al ser este factor primordial en la organización de los diversos procesos cognitivos y procedimentales para la construcción de conocimientos y aprendizajes. En consecuencia, es necesario proseguir en el trabajo de la gamificación como variable motivadora (MeloSolarte \& Díaz, 2018), incentivándose el uso apropiado de las TIC, y la profundización en la comprensión del enfoque centrado en el estudiante (López-de-la-Serna, \& Garrido, 2018).

De la misma manera, se piensa que esta correlación gamificación-aprendizaje autoregulado contribuye a reconocer la importancia de trabajar desde un contexto basado en el florecimiento de aptitudes reflexivas ante la búsqueda confiable de información, resultando primordial en este sentido, la investigación como recurso estratégico para la construcción de aprendizajes y conocimientos. En esta esfera, el aprendizaje autorregulado permite a los estudiantes examinar sus propias acciones, sentimientos, en función de trabajar para ser mejor persona y por ende estudiante (McPherson, et al. 2019). Por lo que se puede concretar que la gamificación contribuye en propiciar la motivación del estudiante no solo hacia el crecimiento académico sino también personal (Buckley, et al., 2018). 
Alex Paúl Zambrano-Álava; Ana Thalía Lucas-Zambrano; María De Los Ángeles Lucas-Zambrano; Karina Elizabeth Luque-Alcívar

\section{CONCLUSIÓN}

Para concluir, se determina que la gamificación se relaciona positivamente con el aprendizaje autorregulado, lo cual favorece al estudiante en su motivación y crecimiento personal como factores esenciales para un rendimiento académico. Asimismo, promueve el aprendizaje centrado en el estudiante. Para ello, tanto docentes como estudiantes se constituyen en equipo cooperativo de trabajo, mediante la figura de asesor y asesorado, valiéndose de la investigación como medio de gestión del conocimiento mediante la autorreflexión como parte del proceso educativo.

\section{REFERENCIAS}

Alemán-Marichal, B, Navarro-de-Armas, O, Suárez-Díaz, R, Izquierdo-Barceló, Y, \& Encinas-Alemán, T. (2018). La motivación en el contexto del proceso enseñanzaaprendizaje en carreras de las Ciencias Médicas. [Motivation in the context of the teaching-learning process in specialties of the Medical Sciences]. RevistaMédicaElectrónica, 40(4), 1257-1270.

Area-Moreira, M., \& González-González, C. S. (2015). De la enseñanza con libros de texto al aprendizaje en espacios online gamificados. [From teaching with textbooks to learning in gamified online spaces]. Educatio Siglo XXI, 33(3 Noviembr), 15-38. https://doi.org/10.6018/j/240791

Buckley, J., DeWille, T., Exton, C., Exton, G., \& Murray, L. (2018). A GamificationMotivation Design Framework for Educational Software Developers. Journal of Educational Technology Systems, 47(1), 101-127. https://doi.org/10.1177/0047239518783153

Castelló, A, \&Cladellas, R. (2013). La evaluación de la comprensión en el aprendizaje: EI empleo de las TIC en el análisis de estructuras de conocimiento. [The assessment of comprehension in learning: ICT in the knowledge structures' analysis]. Estudiospedagógicos (Valdivia), 39(Especial), https://dx.doi.org/10.4067/S0718-07052013000300004 
Alex Paúl Zambrano-Álava; Ana Thalía Lucas-Zambrano; María De Los Ángeles Lucas-Zambrano; Karina Elizabeth Luque-Alcívar

Cortez-Godinez, R. (2017). Un minuto para matemáticas. Una experiencia de diversión, aprendizaje y divulgación al explorar patrones numéricos. [A minute for Mathematics". An Experience of Fun, Learning and Outreach Through the Exploration of Numeric Patterns].Educación matemática, 29(3), 225-243. https://dx.doi.org/10.24844/em2903.08

Daura, F. (2013). El contexto como factor del aprendizaje autorregulado en la educación superior. [Context as a Factor of Self-Regulated Learning in Higher Education]. Educación y Educadores, 16(1), 109-125.

Fernández, A., Olmos, J., \& Alegre, J. (2016). Pedagogical value of a common knowledge repository for Business Management courses. @ tic revista d'innovació educativa(16), 39-47. https://doi.org/10.7203/attic.16.8044

Hernández-Jaime, J, Jiménez-Galán, Y, \& Rodríguez-Flores, E. (2018). Desarrollo de competencias de pensamiento creativo y práctico para iniciar un plan de negocio: diseño de evidencias de aprendizaje. [Developing creative and practical thinking competencies for a business plan: designing learning evidences]. RIDE. Revista Iberoamericana para la Investigación y el Desarrollo Educativo, 9(17), 314-342. https://dx.doi.org/10.23913/ride.v9i17.383

Hernández-Horta, I., Monroy-Reza, A, \& Jiménez-García, M. (2018). Aprendizaje mediante Juegos basados en Principios de Gamificación en Instituciones de Educación Superior. [Learning through Games based on Principles of Gamification in Higher Education Institutions]. Formación universitaria, 11(5), 31-40. https://dx.doi.org/10.4067/S0718-50062018000500031

Hernández-Sampieri, R., Fernández-Collado, C., \& Baptista-Lucio, P. (2014). Metodología de la investigación $6^{\circ}$ edición [Research Methodology 6th edition]. México: McGraw-Hill/Interamericana editores, S.A. de C. V.

Hernández, A. (2018). Transitando por el camino de la escuela para padres, madres y representantes. Unaexperienciavivida. [Walking on the way to school for parents and representatives. A lived experience]. EPISTEME KOINONIA, 1(1), 51-71. http://dx.doi.org/10.35381/e.k.v1i1.490 
Alex Paúl Zambrano-Álava; Ana Thalía Lucas-Zambrano; María De Los Ángeles Lucas-Zambrano; Karina Elizabeth Luque-Alcívar

Liberio-Ambuisaca, X. (2019). El uso de las técnicas de gamificación en el aula para desarrollar las habilidades cognitivas de los niños y niñas de 4 a 5 años de Educación Inicial. [The use of gamification techniques in the classroom to develop the cognitive skills of children from 4 to 5 years of Initial Education].Conrado, 15(70), 392-397.

López-de-la-Serna, A., \& Garrido, C. C. (2018). Estudio de las emociones, el aprendizaje autorregulado y la motivación en un curso SPOC. [Study of emotions, selfregulated learning and motivation in a SPOC course]. IJERI: International Journal of Educational Research and Innovation, (10), 299-316.

Martínez-Albán, W., Vallejo-Valdivieso, P., \& Moya-Martínez, M. (2019). Estructuras mentales y aprendizaje autorregulado en generación de aprendizaje significativo. [Mental structures and self-regulated learning in generating meaningful learning]. $\quad$ CIENCIAMATRIA, 6(10), 629-645. https://doi.org/10.35381/cm.v6i10.259

Medina-Ramírez, R, Álamo-Arce, D, Costa, M, \& Rodríguez-de-Castro, F. (2019). Aprendizaje autorregulado: una estrategia para 'enseñar a aprender' en ciencias de la salud. [Self-regulated learning: a strategy to 'teach to learn' in health sciences]. FEM: Revista de la Fundación Educación Médica, 22(1), 5-10.

Melo-Solarte, D, \& Díaz, P.. (2018). El Aprendizaje Afectivo y la Gamificación en Escenarios de Educación Virtual. [Emotional Learning and Gamification in Virtual Education Environments]. Informacióntecnológica, 29(3), 237-248. https://dx.doi.org/10.4067/S0718-07642018000300237

Morales-Salas, R. (2018). La planeación de la enseñanza-aprendizaje, competencia que fortalece el perfil docente. [The planning of the instruction-learning, competence that strengthens the educator profile]. RIDE. Revista Iberoamericana para la Investigación y el Desarrollo Educativo, 8(16), 311-334. https://dx.doi.org/10.23913/ride.v8i16.343

Muñoz-Morales, N., Barrientos-Oradini, N., Araya-Castillo, L., \& Reyes-Saavedra, J. (2019). Capacidades metacognitivas en el sistema educativo en instituciones educativas de educación media. [Metacognitive capacities in the educational system in educational institutions of secondary education]. Revista Arbitrada Interdisciplinaria Koinonía, 4(7), 103-127. http://dx.doi.org/10.35381/r.k.v4i7.196 
Alex Paúl Zambrano-Álava; Ana Thalía Lucas-Zambrano; María De Los Ángeles Lucas-Zambrano; Karina Elizabeth Luque-Alcívar

Muñoz-González, D. (2018). La estimulación cognitiva como estrategia para la atención psicogerontológica a los adultos mayores con demencia. [Cognitive stimulation as a strategy for psychogerontological care for the elderly with dementia]. Revista Cubana de Salud Pública, 44(3), 1-8.

Mujica-Johnson, F, \& Toro-Oyarzún, M. (2019). Formación afectiva en la educación parvularia de Chile. [Affective formation in the Preschool Education of Chile]. Cuadernos de InvestigaciónEducativa, 10(2), 57-71.

McPherson, G. E., Osborne, M. S., Evans, P., \&Miksza, P. (2019). Applying self-regulated learning microanalysis to study musicians' practice. Psychology of Music, 47(1), 18-32. https://doi.org/10.1177/0305735617731614

Núñez, J., David-Álvarez, N., García, T., \&Dobarro, A. (2019). Escala de Evaluación de la Autorregulación del Aprendizaje a partir de Textos (ARATEX-R. [Self-Regulated Learning from Texts (ARATEX-R) Assessment Scale]. Eur. j. educ. psychol. 8(1), 9-22. https://doi.org/10.30552/ejep.v8i1.142

Ortiz-Colón, A., Jordán, J, \&Agredal, M. (2018). Gamificación en educación: una panorámica sobre el estado de la cuestión. [Gamification in education: an overview on the state of the art]. Educação e Pesquisa, 44, e173773. EpubApril 23, 2018.https://doi.org/10.1590/s1678-4634201844173773

Olea-Miranda, J, Contreras, O, \&Barcelo-Valenzuela, M. (2016). Las capacidades de absorción del conocimiento como ventajas competitivas para la inserción de pymes en cadenas globales de valor. Estudios Gerenciales, 32(139),127-136.

Páez, A. (2018). Docentes y padres en el proceso de aprendizaje de los estudiantes. [Teachers and parents in the student learning process]. EPISTEME KOINONIA, 1(2), 18-34. http://dx.doi.org/10.35381/e.k.v1i2.509

Parra-González, M., \& Segura-Robles, A. (2019). Traducción y validación de la escala de evaluación de experiencias gamificadas (GAMEX).[Translation and validation of the Gameful Experience Scale (GAMEX)]. 71 (4), 87-99. https://doi.org/10.13042/Bordon.2019.70783

Peche-Cruz, H., \& Giraldo-Supo, V. (2019). El Aprendizaje Flip Learning centrado en el estudiante como generador de calidad educativa. [Student-centered Flip Learning as a generator of educational quality]. Revista Arbitrada Interdisciplinaria Koinonía, 4(8), 427-450. http://dx.doi.org/10.35381/r.k.v4i8.293 
Alex Paúl Zambrano-Álava; Ana Thalía Lucas-Zambrano; María De Los Ángeles Lucas-Zambrano; Karina Elizabeth Luque-Alcívar

Torrano, F, Fuentes, J, \& Soria, M. (2017). Aprendizaje autorregulado: estado de la cuestión y retos psicopedagógicos. [Self-regulated learning: state of the issue and psycho-pedagogical challenges]. Perfiles educativos, 39(156), 160-173.

Valda-Sánchez, F, \& Arteaga-Rivero, C. (2015). Diseño e implementación de una estrategia de gamificacion en una plataforma virtual de educación. [Design and implementation of a gamification strategy in a virtual education platform]. Fides et Ratio - Revista de Difusión cultural y científica de la Universidad La Salle en Bolivia, 9(9), 65-80.

Valenzuela, J, Muñoz-Valenzuela, C, Silva-Peña, I, Gómez-Nocetti, V, \&PrechtGandarillas, A. (2015). Motivación escolar: Claves para la formación motivacional de futuros docentes. [School motivation: Keys to motivational training for future teachers].Estudiospedagógicos (Valdivia), 41(1), 351-361. https://dx.doi.org/10.4067/S0718-07052015000100021

Vallejo-Valdivieso, P., Zambrano-Pincay, G., Vallejo-Pilligua, P., \& Bravo-Cedeño, G. (2019). Modelos de planificación educativa y diversidad en aulas de clases. [Models of educational planning and diversity in classrooms]. CIENCIAMATRIA, 5(9), 302-315. https://doi.org/10.35381/cm.v6i10.149

Villarroel, V, \&Bruna, D. (2017). Competencias Pedagógicas que Caracterizan a un Docente Universitario de Excelencia: Un Estudio de Caso que Incorpora la Perspectiva de Docentes y Estudiantes. [Pedagogical Competencies of University Teachers: A Case Study, Which Incorporates the Perspective of Chilean Teachers and Students]. Formación universitaria, 10(4), 75-96. https://dx.doi.org/10.4067/S0718-50062017000400008

Zambrano-Pincay, G., Vallejo-Valdivieso, P., Vallejo-Pilligua, P., \& Bravo-Cedeño, G. (2019). Los profesionales de la Psicopedagogía en la atención a la diversidad como Agente Educativo. [Professionals of Psychopedagogy in attention to diversity as an Educational Agent]. Revista Arbitrada Interdisciplinaria de Ciencias de la Salud. Salud y Vida, 3(6), 41-57. http://dx.doi.org/10.35381/s.v.v3i6.304

Zepeda-Hernández, S, Abascal-Mena, R, \& López-Ornelas, E. (2016). Integración de gamificación y aprendizaje activo en el aula. [Integration of gamification and active learning in theclassroom]. Ra Ximhai, 12(6),315-325. 
EPISTEME KOINONIA

Revista Electrónica de Ciencias de la Educación, Humanidades, Artes y Bellas Artes

Año III. Vol III. N5. Enero - Junio, 2020

Hecho el depósito de Ley: FA2018000022

ISSN: 2665-0282

FUNDACIÓN KOINONIA (F.K).

Santa Ana de Coro, Venezuela

Alex Paúl Zambrano-Álava; Ana Thalía Lucas-Zambrano; María De Los Ángeles Lucas-Zambrano; Karina Elizabeth Luque-Alcívar

C2020 por el autor. Este artículo es de acceso abierto y distribuido según los términos y condiciones de la licencia CreativeCommons Atribución-NoComercial-Compartirlgual 4.0 Internacional (CC BY-NC-SA 4.0)

(https://creativecommons.org/licenses/by-nc-sa/4.0/). 